\title{
On Inner Characterizations of Pseudo-Krein and Pre-Krein Spaces
}

\author{
By \\ Gerald HofmanN*
}

\begin{abstract}
For developing a theory of *-representations of *-algebras $\mathcal{A}$ on (indefinite) inner product spaces $E,(\cdot, \cdot)$, the classes of pseudo-Krein and pre-Krein spaces which admit a Hilbert space structure and exactly one maximal Hilbert space structure, respectively, are introduced. Inner characterizations for the topological structure of $E,(\cdot, \cdot)$ to be a pseudo-Krein and pre-Krein space, respectively, are given. The results are illustrated by some examples.
\end{abstract}

\section{$\S 1 . \quad$ Introduction}

In order to extend the Borchers-Uhlmann approach to General (axiomatic) QFT such that it applies to some more models (e.g., gauge fields ([9, Chapter 10], [19], [25], [13], [14]), massless fields ([30], [21]), Euclidean random fields $([1],[2]))$, the GNS (Gelfand, Neumark, Segal) representation ([26, Chapter 17.4, Theorem 2]) had to be generalized. In contrast to the well-known GNS representation, a hermitian linear functional (pseudo-Wightman functional) $W$ has now to be considered on a unital *-algebra $\mathcal{A}$. The GNS construction then gives a representation of $\mathcal{A}$ on a (possibly indefinite) inner product space $\mathcal{D},(\cdot, \cdot)$ (see [3], [15], [21], [36]). Among others, the following new features enter the theory in contrast to the well-known reconstruction theorem for positive functionals:

(a) A priori, the (common and invariant) domain $\mathcal{D}$ of the operators of the representation $\pi_{W}(\cdot)$ does not carry any scalar product $[\cdot, \cdot \cdot]$ such that the

Communicated by T. Kawa. Received June 14, 2001. Revised December 21, 2001.

2000 Mathematics Subject Classification(s): 46C20, 46K10.

*Staatl. Studienakademie, Am Wartenberg 2, D-99817 Eisenach, Germany. 
indefinite metric $(\cdot, \cdot)$ inherited by $W$ on $\mathcal{D}$ satisfies $(\cdot, \cdot)=[\cdot, J$.$] , where$ $J$ is a bounded Gram-operator. However, for mathematically (see [15]) as well as physically (see [16, Chapter 3]) motivated reasons, it is desirable to introduce such a Hilbert space structure $(\mathcal{H}, J)$ on $\mathcal{D}$, where $\mathcal{H}=\widetilde{\mathcal{D}}^{\tau(\|\cdot\|)}$, (completed hull relative to $\tau(\|\cdot\|),\|\cdot\|=\sqrt{[\cdot, \cdot]}$ ).

(b) If there are Hilbert-space structures on $\mathcal{D}$, then even the maximal one (see [15, Proposition 2]) is not uniquely defined in general. Let us mention that a whole family of non-equivalent maximal Hilbert-space structures was explicitly constructed by Araki ([4]) in the case of $\mathcal{A}=\left(\mathbb{C}^{2}\right)_{\otimes}($ tensor algebra over $\mathbb{C}^{2}$ ).

While group representations on (indefinite) inner product spaces $E,(\cdot, \cdot)$ were considered by Araki in [5], the aim of the present paper is to start a general theory of $*$-representations of *-algebras $\mathcal{A}$ on inner product spaces.

Since on one hand there are totally discontinuous inner product spaces (for examples, see [6, p. 36], [8, Example III.3.2]) and on the other hand, as explained in (a), one would like to introduce a Hilbert space structure on the representation space, it is obvious that not every inner product space $E,(\cdot, \cdot)$ is suitable for being a representation space. As a first step towards a representation theory of *-algebras on inner product spaces, those inner product spaces which are suitable for being representation space are singled out.

Along these lines, in Definition 2.1 the class of pseudo-Krein spaces consisting of those inner product spaces which allow a Hilbert space structure is introduced. According to (b) given above, those pseudo-Krein spaces on which exactly one (up to isometric linear isomorphisms) maximal Hilbert space structure exists are collected in the subclass of pre-Krein spaces.

The present paper is aimed at an investigation of the classes of pseudoKrein and pre-Krein spaces, especially at their inner characterizations. For achieving that goal, the topological structure of the inner product space $E,(\cdot, \cdot)$ has to be considered. Taking the algebraic structure of $E,(\cdot, \cdot)$ into account, there are two strategies to introduce topologies on $E$ :

1) Considering the dual pairing $(E, E)$, where the pairing is given by the inner product $(\cdot, \cdot)$, the following locally convex (l.c.) topologies are defined on $E$ : the weak, Mackey and strong topology, and furthermore, the topology of uniform convergence on strongly bounded subsets.

2) Considering continuity properties of the inner product $(\cdot, \cdot)$, the notion of majorant and partial majorant toplogies follows. 
Among others, it then follows: An inner product space $E,(\cdot, \cdot)$ is a pseudoKrein space if, and only if, there exists a quadratic (Hilbertian) norm $q$ on $E$ such that the topology defined by $q$ is finer than the weak topology of the dual pairing considered in 1) (see Theorem 4.1). In Theorem 5.1 it is stated that a pseudo-Krein space $E,(\cdot, \cdot)$ is a pre-Krein space if, and only if, all minimal majorants defined by a quadratic norm are equivalent on $E$. For applications, convenient criteria such that a pseudo-Krein space is pre-Krein follow from Theorem 5.1. E.g., if $E,(\cdot, \cdot)$ is a pseudo-Krein space and the dual pair $(E, E)$ is reflexive, then it is a pre-Krein space (see Corollary 5.1).

If the inner product space $E,(\cdot, \cdot)$ under consideration is decomposable, much more can be shown. First of all, every decomposable inner product space is a pseudo-Krein space (Corollary 4.1). Furthermore, a decomposable inner product space $E,(\cdot, \cdot)$ is pre-Krein if, and only if, there exists a fundamendal decomposition $E=E^{(+)}(\dot{+}) E^{(-)}$such that $E^{(+)}$or $E^{(-)}$is complete relative to its intrinsic topology (Proposition 5.1). Let us mention that the question whether or not a pseudo-Krein space is pre-Krein is treated, using different methods, in [11].

The pattern of the present paper is as follows. In Section 2 the preliminaries from the theory of inner product spaces are recalled. According to 1) and 2), topologies are introduced on $E$ in Sections 3.1 and 3.2, respectively. While pseudo-Krein spaces are investigated in Section 4, the subclass of pre-Krein spaces is studied in Section 5. Finally, in Section 6 examples are considered for illustrating our results.

\section{$\S 2 . \quad$ Some Preliminaries on Inner Product Spaces}

The following considerations are concerned with inner product spaces $E,(\cdot, \cdot)$, where $E$ denotes a (complex) vector space and $(\cdot, \cdot)$ an inner product on $E$, i.e., $(\cdot, \cdot): E \times E \rightarrow \mathbb{C}$ is a hermitian sesquilinear form $\left(\left(x, c_{1} y_{1}+c_{2} y_{2}\right)=\right.$ $\left.c_{1}\left(x, y_{1}\right)+c_{2}\left(x, y_{2}\right),(x, y)=\overline{(y, x)}, x, y, y_{j} \in E, c_{j} \in \mathbb{C}, j=1,2\right)$. Two inner product spaces $E_{j},(\cdot, \cdot)_{j}, j=1,2$, will be identified in the following, if they are isometrically isomorphic, (i.e., there is a linear bijection $\psi: E_{1} \rightarrow E_{2}$ satisfying $\left.(x, y)_{1}=(\psi(x), \psi(y))_{2}, x, y \in E_{1}\right)$. This will be denoted by $E_{1} \equiv E_{2}$.

Throughout the present paper let us assume that $E,(\cdot, \cdot)$ is non-degenerate, i.e., $(x, y)=0$ for all $y \in E$ implies $x=0$. That assumption is no significant restriction since for every degenerate inner product space $E,(\cdot, \cdot)$, there is a non-degerate one $\hat{E}=E / E^{(0)}$ (quotient space) endowed with the inner product $(\hat{x}, \hat{y})^{\sim}=(x, y), x, y \in E, \hat{x}=x+E^{(0)}, \hat{y}=y+E^{(0)} \in \hat{E}$, such that $E \equiv \hat{E}(\dot{+})$ 
$E^{(0)}$, where

$$
E^{(0)}=\{x \in E ;(x, y)=0 \text { for all } y \in E\}
$$

denotes the isotropic part of $E$, and $(\dot{+})$ denotes the orthogonal direct sum (see $[7],[8])$.

Noticing $(x, x) \in \mathbb{R}$ for each $x \in E$, a vector $x_{0} \in E$ is called positive (neutral and negative, respectively), if $\left(x_{0}, x_{0}\right)>0\left(\left(x_{0}, x_{0}\right)=0\right.$ and $\left(x_{0}, x_{0}\right)<$ 0 , respectively) hold. Recall the following notations taken from [7], [8].

$\mathcal{P}^{0}(E):=\{x \in E ;(x, x)=0\}$ denotes the set of all neutral vectors of $E$ (the neutral part of $E)$,

$\mathcal{P}^{++}(E):=\{x \in E ;(x, x)>0\} \cup\{0\}$ denotes the set of all positive vectors of $E$ (the positive part of $E$ ),

$\mathcal{P}^{--}(E):=\{x \in E ;(x, x)<0\} \cup\{0\}$ denotes the set of all negative vectors of $E$ (the negative part of $E$ ),

If it is clear from the context, then let us write $\mathcal{P}^{x}$ instead of $\mathcal{P}^{x}(E), x \in$ $\{0,++,--\}$, in the following. Obviously, $E=\mathcal{P}^{++} \cup \mathcal{P}^{--} \cup \mathcal{P}^{0}$.

Recall further that a nondegenerate inner product space $E,(\cdot, \cdot)$ is called decomposable, if it admits a fundamental decomposition

$$
E=E^{(+)}(\dot{+}) E^{(-)},
$$

where $E^{( \pm)} \subset \mathcal{P}^{ \pm \pm}$, and since $(\dot{+})$ denotes the orthogonal direct sum, it holds $E^{(+)} \cap E^{(-)}=\{0\}$, and $x \in E^{(+)}, y \in E^{(-)}$imply $(x, y)=0$.

For every definite linear subspace $L \subset \mathcal{P}^{ \pm \pm}$, the intrinsic topology $\tau_{\text {int }}(L)$ is defined by the norm $x \rightarrow \sqrt{|(x, x)|}, x \in L$. Remember that an inner product space $\mathfrak{K},(\cdot, \cdot)$ is called a Krein space, if it is decomposable with fundamental decomposition

$$
\mathfrak{K}=\mathfrak{K}^{(+)}(\dot{+}) \mathfrak{K}^{(-)},
$$

and $\mathfrak{K}^{( \pm)} \subset \mathcal{P}^{ \pm \pm}$are $\tau_{\text {int }}\left(\mathfrak{K}^{( \pm)}\right)$-complete. Recall also that $\mathfrak{K},(\cdot, \cdot)$ is a Krein space if, and only if, there are a (positive definite) scalar product $[\cdot, \cdot]$ turning $\mathfrak{K}$ into a Hilbert space as well as some symmetry $J: \mathfrak{K} \rightarrow \mathfrak{K}$ (i.e., $J=J^{-1}=$ $J^{[*]}, J^{[*]}$ denotes the Hilbert space adjoint) such that $(x, y)=[x, J y], x, y \in \mathfrak{K}$. Considering the norm $x \rightarrow\|x\|:=\sqrt{[x, x]}, x \in \mathfrak{K}$, let $\tau=\tau(\|\cdot\|)$ denote the l.c. topology defined by $\|\cdot\|$ on $\mathfrak{K}$. $\tau$ is called the canonic topology of the Krein space $\mathfrak{K},(\cdot, \cdot)$. Notice that $\tau$ does not depend on the special choice of the fundamental decomposition (1).

We are now prepared for giving the following definition. 
Definition 2.1. Let $E,(\cdot, \cdot)$ be a non-degenerate inner product space.

a) $E,(\cdot, \cdot)$ is then called a pseudo-Krein space, if there is some Krein space $\mathfrak{K},(\cdot, \cdot) \sim$ such that $E$ is densely embedded in $\mathfrak{K}$ relative to $\tau=\tau(\|\cdot\|)$, and $(x, y)=(x, y) \sim$ for each $x, y \in E$.

b) A pseudo-Krein space is called a pre-Krein space, if there is exactly one Krein space such that a) applies.

Remark.

a) $E,(\cdot, \cdot)$ is a pseudo-Krein space if, and only if, a Hilbert space structure exists on $E,(\cdot, \cdot)$ (see $[15$, Theorem 3 (iii) $\Leftrightarrow$ (iv)]).

b) Krein spaces and pre-Hilbert spaces obviously are special instances of preKrein spaces.

Let us finally confirm that . $\uparrow_{E}$ denotes the restriction of a norm, a topology and an inner product onto a linear subspace $E$.

\section{$\S 3 . \quad$ Topologies on Inner Product Spaces}

\section{$\S 3.1$. Dual pairings with inner product spaces}

Recall that a dual pairing is a triplet (!) consisting of two vector spaces $E, F$ and a non-degenerate bilinear form $b: E \times F \rightarrow \mathbb{C}$, and notice that the replacement of $b$ by a non-degenrate inner product $(\cdot, \cdot)$ does not have any impact at the following considerations. For a non-degenerate inner product space $E,(\cdot, \cdot)$, let us consider the dual pairing $(E, E)$ with the inner product $(\cdot, \cdot)$ in the following.

Let $\mathfrak{P}(\tau)$ denote a system of seminorms defining a locally convex (l.c.) topology $\tau$ on $E$ ([18, Section 18.1]). Remember the following l.c. topologies defined by the above dual pairing ([17, Chapter 8.4$])$ :

a) the weak topology $\sigma(E, E)$ defined by

$$
\mathfrak{P}(\sigma)=\left\{f \rightarrow p_{\mathcal{F}}(f):=\sup _{\varphi \in \mathcal{F}}|(f, \varphi)| ; \mathcal{F} \in \mathfrak{F}\right\},
$$

$f \in E$, and $\mathfrak{F}$ denotes the set of alle finite subsets of $E$.

b) the strong topology $\beta(E, E)$ defined by

$$
\mathfrak{P}(\beta)=\left\{f \rightarrow p_{\mathcal{B}}(f):=\sup _{\varphi \in \mathcal{B}}(|(f, \varphi)|) ; \mathcal{B} \in \mathfrak{B}\right\},
$$

where $\mathfrak{B}$ denotes the set of all $\sigma$-bounded subsets of $E$, 
c) the topology of uniform convergence on strongly bounded subsets denoted by $\beta^{*}(E, E)$ and defined by

$$
\mathfrak{P}\left(\beta^{*}\right)=\left\{f \rightarrow p_{\mathcal{D}}(f):=\sup _{\varphi \in \mathcal{D}}(|(f, \varphi)|) ; \mathcal{D} \in \mathfrak{D}\right\},
$$

where $\mathfrak{D}$ denotes the set of all $\beta$-bounded subsets of $E$,

d) the Mackey topology $\mu(E, E)$ defined by

$$
\mathfrak{P}(\mu)=\left\{f \rightarrow p_{\mathcal{C}}(f):=\sup _{\varphi \in \mathcal{C}}(|(f, \varphi)|) ; \mathcal{C} \in \mathfrak{C}\right\},
$$

where $\mathfrak{C}$ denotes the set of all absolutely convex and $\sigma(E, E)$-compact subsets of $E$.

If it is clear from the context, then the above topologies are correspondingly written $\sigma, \beta, \beta^{*}, \mu$. About two l.c. topologies $\tau_{1}, \tau_{2}$, let $\tau_{1} \prec \tau_{2}$ (resp. $\tau_{1} \supsetneqq \tau_{2}$ ) denote the relation that $\tau_{1}$ is weaker (coarser) than $\tau_{2}$ (resp. $\tau_{1}$ is strictly weaker than $\tau_{2}$ ). Furthermore, $\tau_{1}=\tau_{2}$ says that topologies $\tau_{1}$ and $\tau_{2}$ are equivalent. Recall then that

$$
\sigma \prec \mu \prec \beta^{*} \prec \beta \text {. }
$$

Remember also that if $\mathfrak{K},(\cdot, \cdot)$ is a Krein space, then its canonical topology $\tau$ introduced above satisfies $\tau=\mu=\beta$.

Concerning reflexivity and semireflexivity of the above dual pairing $(E, E)$, the following proposition holds, and will be used in Section 5 .

Proposition 3.1. About the dual pairing $(E, E)$ with inner product $(\cdot, \cdot)$, the following are equivalent:

(i) $(E, E)$ is reflexive,

(ii) $(E, E)$ is semireflexive,

(iii) $E[\mu]$ is barrelled,

(iv) $\mu=\beta$,

(v) $E[\sigma]$ is quasicomplete.

Proof. See [33, Theorems 10.2.4 and 10.3.2]. 


\section{$\S 3.2$. On the continuity of the inner product}

Concerning continuity properties of the inner product $(\cdot, \cdot)$, recall that a l.c. topology $\tau$ defined on $E$ is called majorant (resp. partial majorant), if the inner product is (jointly) continuous (resp. separately continuous) relative to $\tau$. For every norm $p$ on $E, \tau(p)$ denotes the l.c. topology defined by $p$. Obviously, if $\tau$ is a majorant on $E,(\cdot, \cdot)$, then there is a norm $p$ on $E$ such that $\tau \succ \tau(p)$. Using now the weak topology $\sigma$ introduced above, we have the following characterization of majorants and partial majorants, respectively.

Proposition 3.2. Let $E,(\cdot, \cdot)$ be a non-degenerate inner product space.

a) A l.c. topology $\tau$ defined on $E$ is a partial majorant if and only if $\sigma \prec \tau$.

b) The following are equivalent:

(i) a majorant exists on $E,(\cdot, \cdot)$,

(ii) a metrizable l.c. topology $\rho$ exists on $E$ such that $\sigma \prec \rho$,

(iii) a norm $p$ exists on $E$ such that $\sigma \prec \tau(p)$,

(iv) a norm $q$ exists on $E$ such that $|(x, y)| \leq q(x) q(y)$ for all $x, y \in E$.

Proof. a) See [8, Theorem III.2.1]. b) See [8, Theorem IV.2.1, Lemma IV.1.2].

Remark. While partial majorants always exist due to Proposition 3.2 a), there are examples of inner product spaces without any majorant (see $[6$, p. 36], [8, Example III.3.2]).

For every norm $p$ on $E$, consider

$$
p^{\prime}(x)=\sup _{0 \neq y \in E} \frac{|(x, y)|}{p(y)}=\sup _{p(y) \leq 1}|(x, y)| .
$$

Notice that $0<p^{\prime}(x)<\infty$ for each $0 \neq x \in E$ if, and only if, $\sigma \prec \tau(p)$. $p^{\prime}$ is then called the polar norm relative to $p$. If $p=p^{\prime}$, then $p$ is called self-polar.

For every norm $p$, let $\mathcal{B}_{p}=\{x \in E ; p(x) \leq 1\}$ denote the unit-ball relative to $p$. Furthermore, let $\mathfrak{T}$ denote the set of all normed majorants $\tau(p)$ on $E,(\cdot, \cdot)$. The following technical lemma then applies.

Lemma 3.1. Let $E,(\cdot, \cdot)$ be a non-degenerate inner product space and $p$ a norm on $E$. The following hold. 
a) $\tau(p) \in \mathfrak{T}$ if, and only if, $\tau\left(p^{\prime}\right) \prec \tau(p)$.

b) If $\tau(p) \in \mathfrak{T}$, then

(i) $\mathcal{B}_{p}$ is $\sigma$-bounded,

(ii) $\tau\left(p^{\prime}\right) \prec \beta$,

(iii) $\beta^{*} \prec \tau(p)$.

Proof. The proof uses some ideas taken from [35]. a) $(\Rightarrow)$ : Assuming that $\tau(p) \in \mathfrak{T}$, there is a constant $0<c<\infty$ such that

$$
|(x, y)| \leq c p(x) p(y),
$$

$x, y \in E . \quad(3)$ now implies $p^{\prime}(x)=\sup _{y \in \mathcal{B}_{p}}|(x, y)| \leq c p(x), x \in E$, and consequently $\tau\left(p^{\prime}\right) \prec \tau(p)$. $(\Leftarrow)$ : Assuming $\tau\left(p^{\prime}\right) \prec \tau(p)$, there is a constant $0<d<\infty$ such that $p^{\prime}(x) \leq d p(x), x \in E$. Now (2) yields

$$
|(x, y)| \leq p^{\prime}(x) p(y) \leq d p(x) p(y)
$$

for each $x, y \in E$. Hence, $\tau(p) \in \mathfrak{T}$.

b) (i): Take $\mathcal{F}=\left\{\varphi_{1}, \ldots, \varphi_{n}\right\} \in \mathfrak{F}, n \in \mathbb{N}$, and notice that for each $y \in \mathcal{B}_{p}$, $p(y) \leq 1$ and

$$
\begin{aligned}
p_{\mathcal{F}}(y) & =\max _{j=1, \ldots, n}\left|\left(y, \varphi_{j}\right)\right| \leq c \max _{j=1, \ldots, n} p\left(\varphi_{j}\right) p(y) \\
& \leq c \max _{j=1, \ldots, n} p\left(\varphi_{j}\right)
\end{aligned}
$$

follow from (3). Noticing that the right hand side of the above chain of inequalities does not depend on $y$, (i) follows. (ii): Since $\mathcal{B}_{p}$ is $\sigma$-bounded by (i), it follows that $x \rightarrow \sup _{y \in \mathcal{B}_{p}}\{|(x, y)|\}, x \in E$, is a $\beta$-continuous norm on $E$. Due to $(2), \tau\left(p^{\prime}\right) \prec \beta$ follows. (iii): Let $\mathcal{D}$ be any $\beta$-bounded subset of $E$, i.e., for every $\sigma$-bounded subset $\mathcal{B} \subset E$ there is some constant $c>0$ depending on $\mathcal{D}$ and $\mathcal{B}$ such that

$$
\sup _{x \in \mathcal{B}} \sup _{y \in \mathcal{D}}|(x, y)| \leq c .
$$

Considering especially $\mathcal{B}=\mathcal{B}_{p}$, we obtain $\sup _{p(x) \leq 1} \sup _{y \in \mathcal{D}}|(x, y)| \leq c$, and consequently

$$
\sup _{y \in \mathcal{D}}|(x, y)| \leq c p(x)
$$

for each $x \in E$. Recalling the above definition of $\mathfrak{P}\left(\beta^{*}\right), \beta^{*} \prec \tau(p)$ follows.

Consider now the above semi-ordering “ $\prec$ ” on $\mathfrak{T}$. A majorant $\tau_{*} \in \mathfrak{T}$ is then called minimal, if there is no $\tau \in \mathfrak{T}$ satisfying $\tau \supsetneqq \tau_{*}$. Furthermore, if 
there is some $\tau^{*} \in \mathfrak{T}$ such that $\tau^{*} \prec \tau$ for every $\tau \in \mathfrak{T}$, then $\tau^{*}$ is called the weakest majorant.

Recalling that $\tau \in \mathfrak{T}$ is minimal if, and only if, there is a norm $p$ on $E$ such that $\tau(p)=\tau\left(p^{\prime}\right)$ (see [8, Theorem IV.4.2]), we obtain readily the following from Lemma 3.1.

Corollary 3.1. If $\tau \in \mathfrak{T}$ is a minimal majorant on $E,(\cdot, \cdot)$, then $\beta^{*} \prec$ $\tau \prec \beta$.

If $\mathfrak{T} \neq \emptyset$, then the existence of minimal majorants follows from the interesting Aronszajn-Schatten construction of self-polar norms ([8, Section IV.4], [6, Theorem 3]) briefly recalled now. Assuming that $\tau(p) \in \mathfrak{T}$, define recursively

$$
p^{(n+1)}(x)=\sqrt{\frac{1}{2}\left(\left(p^{(n)}(x)\right)^{2}+\left(p^{(n)^{\prime}}(x)\right)^{2}\right)},
$$

$p^{(1)}=p, n \in \mathbb{N}$. It then follows that

$$
\widetilde{p}(x)=\lim _{n \rightarrow \infty} p^{(n)}(x),
$$

$x \in E$, is a norm on $E$ satisfying (i) $\tau(\widetilde{p}) \prec \tau(p)$ and (ii) $\widetilde{p}=\widetilde{p}^{\prime}$. Due to Lemma 1a) and [8, Theorem IV.4.2], $\tau(\widetilde{p})$ is a minimal majorant. Recall also that $\tau(p)=\tau(q) \in \mathfrak{T}$ implies $\tau(\widetilde{p})=\tau(\widetilde{q})$ (see [35, Satz 1, Korollar]).

Remember that, a norm and a seminorm $p$ are called quadratic (or Hilbertian) if there is a scalar product and semi-scalar product $[\cdot, \cdot]$, respectively, defined on $E$ such that $p(x)=\sqrt{[x, x]}, x \in E$. A topology $\tau$ on $E$ is then called quadratic, if there is a system of quadratic seminorms $\left\{p_{\alpha}\right\}_{\alpha \in A}$ defining $\tau$ (here $A$ is a set of indices). Recall further that if the norm $p$ considered prior to (4) is quadratic, then all the $p^{(n)}, n \in \mathbb{N}$, considered in (4) and $\widetilde{p}$ are quadratic, too (see [15, Lemma 1]).

The Aronszajn-Schatten construction, Corollary 3.1 and Proposition 3.1 readily yield the following sufficient criterion for the existence of $\tau^{*}$.

Corollary 3.2. If a majorant exists on an inner product space $E,(\cdot, \cdot)$ and one (and consequently all) of the properties listed in Proposition 1 apply, then the weakest majorant $\tau^{*}$ exists on $E,(\cdot, \cdot)$.

In order to decide whether or not the weakest majorant $\tau^{*}$ exists on $E,(\cdot, \cdot)$, the Nevanlinna-pseudometric $\eta([27])$ is a useful prerequisite. Considering the quadratic form

$$
Q(x)=(x, x),
$$

$x \in E$, remember the following. 
Definition 3.1. Let $E,(\cdot, \cdot)$ be a (non-degenerate) inner product space. The Nevanlinna-pseudometric $\eta$ is defined by the system of neighborhoods

$$
\mathfrak{W}_{x}=\left\{U_{x}(\varepsilon) \cap V_{x}\left(q, \varepsilon^{\prime}\right) ; U_{x}(\varepsilon) \in \mathfrak{U}_{x}, V_{x}\left(q, \varepsilon^{\prime}\right) \in \mathfrak{V}_{x}\right\}
$$

of $x \in E$, where $\mathfrak{U}_{x}=\left\{U_{x}(\varepsilon)=\{y \in E ;|Q(y)-Q(x)|<\varepsilon\} ; \varepsilon>0\right\}, \mathfrak{V}_{x}=$ $\left\{V_{x}(q, \varepsilon)=\{y \in E ; q(x-y)<\varepsilon\} ; q \in \mathfrak{P}(\sigma), \varepsilon>0\right\}$.

Remark.

a) It is obvious by definition that $\sigma \prec \eta$.

b) In general, $\eta$ is not a vector space topology, since the vector space operation "+" is not necessarily continuous relative to $\eta$.

For the sequal let us recall the following. The form $Q$ is lower semicontinuous relative to some vector space topology $\tau$, if for every $x_{0} \in E$ and $\gamma<Q\left(x_{0}\right)$ there is a $\tau$-neighborhood $U$ of $x_{0}$ such that $\gamma<Q(y)$ for each $y \in U$ ([18, Section $6.2(2)]$ ). Furthermore, for any two topologies $\tau, \tau^{\prime}$ on $E$ let us consider the weakest topology $\tau \vee \tau^{\prime}$ finer than $\tau$ and $\tau^{\prime}$ ([33, Chapter 1.6.8, 1.6.9]).

The following due to Wittstock ([35, Sätze 5, 6]) and Nevanlinna is known about $\eta$.

Lemma 3.2. Let $E,(\cdot, \cdot)$ be a non-degenerate inner product space.

a) The topology $\eta$ is translationally invariant, and the scalar multiplication is continuous relative to $\eta$.

b) $\eta \prec \tau(p)$ for every $\tau(p) \in \mathfrak{T}$.

c) Under the assumption that $\tau$ is a vector space topology on $E$ satisfying $\sigma \prec \tau$, the following are equivalent:

(i) $\tau \vee \eta$ is a vector space topology on $E$.

(ii) One of the quadratic forms $Q$ or $-Q$ is lower semicontinous relative to $\tau$.

d) If the assertion of c) (ii) is satisfied, then the weakest majorant $\tau^{*}$ exists on $E,(\cdot, \cdot)$, and $\tau^{*}=\left(\beta^{*} \vee \eta\right) \in \mathfrak{T}$.

Proof. a) For showing the translation invariance of $\eta$, take any $x_{0} \in$ $E, \varepsilon>0$. Since $\sigma$ is tranlationally invariant, it is enough to show that for every $U_{x_{0}}(\varepsilon) \in \mathfrak{U}_{x_{0}}$ there are $U_{0}(\delta) \in \mathfrak{U}_{0}$ and $V_{0}\left(q, \delta^{\prime}\right) \in \mathfrak{V}_{0}$ such that

$$
x_{0}+\left(U_{0}(\delta) \cap V_{0}\left(q, \delta^{\prime}\right)\right) \subset U_{x_{0}}(\varepsilon) .
$$


Considering $z \in U_{0}(\delta) \cap V_{0}\left(q, \delta^{\prime}\right)$ and choosing $\delta=\varepsilon / 2, \delta^{\prime}=\varepsilon / 4, q(\cdot)=$ $\left|\left(x_{0}, \cdot\right)\right| \in \mathfrak{P}(\sigma)$, it follows that

$$
\begin{aligned}
\left|Q\left(z+x_{0}\right)-Q\left(x_{0}\right)\right| & =\left|Q(z)-2 \Re\left(x_{0}, z\right)\right| \\
& \leq|Q(z)|+2 q(z) \\
& \leq \delta+2 \delta^{\prime}=\varepsilon .
\end{aligned}
$$

Hence, $x_{0}+z \in U_{x_{0}}(\varepsilon)$, verifying (5) and the translation invariance of $\eta$. The continuity of the scalar multiplication relative to $\eta$ is obvious.

b) Due to a) and $\sigma \prec \tau(p)$, it remains to show that for each $U_{0}(\varepsilon)$ there is a 0-neighborhood $V=\{x \in E ; p(x) \leq \delta\}$ of $\tau(p)$ such that

$$
V \subset U_{0}(\varepsilon) \text {. }
$$

Choosing $\delta=\sqrt{\varepsilon}$, it follows for $x \in V$ that

$$
|Q(x)-Q(0)|=|(x, x)| \leq p(x)^{2} \leq \varepsilon,
$$

and consequently, $x \in U_{0}(\varepsilon)$.

c) See [35, Satz 5].

d) Assuming that one of $Q$ and $-Q$ is lower semicontinuous relative to $\beta^{*}$, c) yields that $\beta^{*} \vee \eta$ is a vector space topology on $E$. Noticing that the inner product $(\cdot, \cdot)$ is jointly continuous relative to $\beta^{*} \vee \eta$, there is a 0 -neighborhood $U$ of $\beta^{*} \vee \eta$ such that $|(x, y)| \leq 1$ for each $x, y \in U$. Considering the absolutely convex hull $\Gamma(U)$ of $U$, it follows that $|(z, w)| \leq 1$ for each $z, w \in \Gamma(U)$. Hence the Minkowski functional

$$
x \rightarrow p(x)=\inf \left\{\rho>0 ; \rho^{-1} \in \Gamma(U)\right\}
$$

satisfies $|(u, v)| \leq p(u) p(v), u, v \in E$, and thus $p$ is a norm and $\tau(p) \in \mathfrak{T}$. Furthermore, the construction of $p$ yields

$$
\tau(p) \prec\left(\beta^{*} \vee \eta\right) .
$$

Noticing further that every $\tau \in \mathfrak{T}$ satisfies $\tau \succ \beta^{*}$ and $\tau \succ \eta$ by Corollary 1 and Lemma $2 \mathrm{~b})$, respectively, it follows that $\tau \succ\left(\beta^{*} \vee \eta\right)$ ([33, Theorem 1.6.8]). Hence, $\tau^{*}=\tau(p)=\left(\beta^{*} \vee \eta\right) \in \mathfrak{T}$, completing the proof.

Remark.

a) Under the assumption that $E,(\cdot, \cdot)$ is decomposable, the converse statement of Lemma $2 \mathrm{~d}$ ) also holds. In that case, the reader is referred to [34, Satz 9] for further conditions equivalent to the existence of $\tau^{*}$ (see also Proposition 3, below). 
b) In order to check lower semicontinuity for $Q$ and $-Q$, respectively, relative to a locally convex topology $\tau$, it is enough to verify it at $x_{0}=0$ due to the translation invariance of $\eta$ stated in Lemma 3.2 a).

\section{$\S 4 . \quad$ On Pseudo-Krein Spaces}

The present section is aimed at an investigation of pseudo-Krein spaces. To begin with, let us consider the interplay between the inner products of a pseudoKrein space $E,(\cdot, \cdot)$ and the Krein spaces in which $E$ is densely embedded.

Lemma 4.1. Let $E,(\cdot, \cdot)$ be a pseudo-Krein space. If $\mathfrak{K}[\tau],(\cdot, \cdot)^{\sim}$ denotes a Krein space such that Definition 1 a) applies, then

a) $\tau \uparrow_{E}$ is a minimal and quadratic majorant on $E,(\cdot, \cdot)$,

b) the $\tau$-continuous extension $(\cdot, \cdot)^{\hat{0}}$ of the inner product $(\cdot, \cdot)$ satisfies $(\cdot, \cdot)^{\sim}=$ $(\cdot, \cdot)^{\wedge}$ on $\mathfrak{K} \times \mathfrak{K}$.

Proof. Noticing that $\tau$ is a minimal and quadratic majorant on $\mathfrak{K}[\tau]$, $(\cdot, \cdot)^{\sim}$, it follows that $\tau \uparrow_{E}$ is a quadratic majorant on $E,(\cdot, \cdot)$ proving the first half of a) and yielding that the $\tau$-continuous extension $(\cdot, \cdot)$ of $(\cdot, \cdot)$ exists on $\mathfrak{K} \times \mathfrak{K}$. Since $\mathfrak{K}=\bar{E}^{\tau}$ (the $\tau$-closed hull of $E$ in $\mathfrak{K}$ ), for each $x, y \in \mathfrak{K}$ there are sequences $\left\{x_{n}\right\}_{n=1}^{\infty},\left\{y_{n}\right\}_{n=1}^{\infty}, x_{n}, y_{n} \in E$ such that $p\left(x-x_{n}\right) \rightarrow 0, p\left(y-y_{n}\right) \rightarrow 0$ as $n \rightarrow \infty$, where $p$ denotes a norm defining $\tau$. Since every convergent sequence is bounded, there is a constant $c>0$ such that $p\left(x_{n}\right)<c, p\left(y_{n}\right)<c, n \in \mathbb{N}$. b) now follows from

$$
\begin{aligned}
& \left|(x, y)^{\sim}-(x, y)\right| \\
& =\mid\left(x-x_{n}, y-y_{n}\right)^{\sim}+\left(x-x_{n}, y_{n}\right)^{\sim}+\left(x_{n}, y-y_{n}\right)^{\sim}+\left(x_{n}, y_{n}\right)^{\sim} \\
& \quad-\left[\left(x-x_{n}, y-y_{n}\right)^{\hat{H}}+\left(x-x_{n}, y_{n}\right)^{\hat{\alpha}}+\left(x_{n}, y-y_{n}\right)^{\hat{}}+\left(x_{n}, y_{n}\right)\right] \mid \\
& \leq 2\left(p\left(x-x_{n}\right) p\left(y-y_{n}\right)+p\left(y_{n}\right) p\left(x-x_{n}\right)+p\left(x_{n}\right) p\left(y-y_{n}\right)\right) \\
& \leq c p\left(x-x_{n}\right)+c(p(x)+c) p\left(y-y_{n}\right) \\
& \rightarrow 0
\end{aligned}
$$

as $n \rightarrow \infty$. Since

$$
\sup _{0 \neq y \in E} \frac{|(x, y)|}{p(y)}=\sup _{0 \neq z \in \mathfrak{K}} \frac{|(x, z)|}{p(z)},
$$

(ii) implies $\left(p \uparrow_{E}\right)^{\prime}=p \uparrow_{E}$ proving the minimality of the majorant $\tau \uparrow_{E}$ on $E,(\cdot, \cdot)$. 
Remark. Remember that if $\tau(p) \in \mathfrak{T}$ is not minimal, then there are examples of non-degenerate inner product spaces $E,(\cdot, \cdot)$ such that the $\tau(p)$ continuously extended inner product is degenerate on $\widetilde{E}^{\tau(p)} \times \widetilde{E}^{\tau(p)}([12])$. However, the continuously extented inner product $(\cdot, \cdot)^{\wedge}$ considered above is non-degenerate on $\mathfrak{K} \times \mathfrak{K}$ due to Lemma $4.1 \mathrm{~b}$ ).

There is the following inner characterization of pseudo-Krein spaces.

Theorem 4.1. Let $E,(\cdot, \cdot)$ be an inner product space. The following are equivalent:

(i) $E,(\cdot, \cdot)$ is a pseudo-Krein space.

(ii) There exists a minimal and quadratic majorant on $E,(\cdot, \cdot)$.

(iii) There exists a quadratic and self-polar norm $q$ on $E,(\cdot, \cdot)$.

(iv) There exists a quadratic norm $q$ on $E$ such that $\sigma \prec \tau(q)$.

Proof. (i) $\Rightarrow$ (ii) follows from Lemma 4.1 a). (ii) $\Rightarrow$ (iii): Assuming that (ii) holds, there is a quadratic norm $p$ such that $\tau(p)$ is a minimal majorant on $E,(\cdot, \cdot)$. The norm $\widetilde{p}$ defined in (6) is self-polar and quadratic, and thus $q=\widetilde{p}$ satisfies (iii). (iii) $\Rightarrow$ (iv): Assuming that (iii) holds, $q$ is a quadratic norm satisfying $\sigma \prec \tau(q)$ by Corollary 3.1. (iv) $\Rightarrow$ (i): Assuming that (iv) holds, the polar norm $q^{\prime}$ exists and is also quadratic ([15, Lemma 1 ii) $\left.]\right)$. Considering the norm

$$
x \rightarrow p^{(1)}(x)=\sqrt{\frac{1}{2}\left((q(x))^{2}+\left(q^{\prime}(x)\right)^{2}\right)},
$$

$x \in E$, we obtain $\tau\left(p^{(1)}\right) \in \mathfrak{T}$. Using the Aronszajn-Schatten construction described in (4), we get a quadratic and selfpolar norm $\widetilde{p}$ on $E,(\cdot, \cdot)$. Considering the completed hull $\mathfrak{K}=\widetilde{E}^{\tau(\tilde{p})}$, let $(\cdot, \cdot) \sim$ and $[\cdot, \cdot] \sim$ denote respectively, the $\tau(\tilde{p})$-continuous extension of the inner products $(\cdot, \cdot)$ and $[\cdot, \cdot]$ onto $\mathfrak{K} \times \mathfrak{K}$, where $\widetilde{p}(z)=\sqrt{[z, z]^{\sim}}, z \in \mathfrak{K}$. For verifying (i), it remains to show that $\mathfrak{K},(\cdot, \cdot)^{\sim}$ is a Krein space. Noticing first that the extented inner product $(\cdot, \cdot)^{\sim}$ is non-degenreate due to $\widetilde{p}=\widetilde{p}^{\prime}$, we use the Riesz-theorem to define the Gram operator $J: \mathfrak{K} \rightarrow \mathfrak{K}$ by

$$
(x, y)^{\sim}=[x, J y]^{\sim}
$$

$x, y \in \mathfrak{K}$. Furthermore, $J$ is invertible and $J=J^{*}$ (adjoint operator in Hilbert space $\left.\mathfrak{K},[\cdot, \cdot]^{\sim}\right)$. Noting then that $J$ and $J^{-1}$ are bounded linear operators due 
to

$$
\begin{aligned}
\widetilde{p}(J y)^{2} & =\left|(J y, y)^{\sim}\right| \leq \widetilde{p}(y) \widetilde{p}(J y), \\
\widetilde{p}\left(J^{-1} w\right) & =\widetilde{p}^{\prime}\left(J^{-1} w\right)=\sup _{0 \neq x \in \mathfrak{K}} \frac{\left|\left(x, J^{-1} w\right)^{\sim}\right|}{\widetilde{p}(x)}=\sup _{0 \neq x \in \mathfrak{K}} \frac{\left|[x, w]^{\sim}\right|}{\widetilde{p}(x)} \\
& \leq \widetilde{p}(w),
\end{aligned}
$$

we define an equivalent scalar product $[x, y]_{1}=[x,|J| y]^{\sim},|J|=\sqrt{J J^{*}}, x, y \in \mathfrak{K}$ on $\mathfrak{K}$. Noticing finally that $J_{1}=J /|J|$ is a symmetry on $\mathfrak{K},[\cdot, \cdot]_{1}$ satisfying $(x, y)^{\sim}=\left[x, J_{1} y\right]_{1}$, we see that $\mathfrak{K},(\cdot, \cdot) \sim$ is a Krein space.

Corollary 4.1. $\quad$ Every non-degenerate and decomposable inner product space is a pseudo-Krein space.

Proof. Assume that $E,(\cdot, \cdot)$ is a non-degenerate and decomposable inner product space. There is a fundamental decomposition $E=E^{(+)}(\dot{+}) E^{(-)}$. Consider the fundamental symmetry $J=P^{+}-P^{-}, P^{ \pm}: E \rightarrow E^{( \pm)}$(fundametal projections). Recall that the decomposition majorant $\tau_{J}=\tau\left(\|\cdot\|_{J}\right)$, $\|x\|_{J}=\sqrt{(x, J x)}, x \in E$, is a quadratic and minimal majorant on $E,(\cdot, \cdot)$ ([8, Theorem IV.6.1]). The corollary under consideration now follows from Theorem 4.1, (ii) $\Rightarrow(\mathrm{i})$.

Remark. (to Theorem 4.1 (iii)): In order to develop a spectral theory of linear operators on inner product spaces, it was remarked by Tomita (see [31], [24], [22], [23]) that quadratic and self-polar norms (called unitary norms by Tomita) play an essential role, too.

If $E,(\cdot, \cdot)$ is a pseudo-Krein space, then there are in general non-equivalent Krein spaces $\mathfrak{K}_{j},(\cdot, \cdot)_{j}, j=1,2$, such that Definition 2.1 a) applies to both of them. It was observed by Araki ([4, Remark 5]) that it then makes sense to define $\mathfrak{K}_{1} \cap \mathfrak{K}_{2}$.

To be more precise, let us recall the following. Let the Krein spaces $\mathfrak{K}_{j},(\cdot, \cdot)_{j}$ be endowed with their canonical topologies $\tau^{(j)}=\tau\left(p_{j}\right)$, where the defining norms satisfy $p_{j}\left(x_{j}\right)=p_{j}{ }^{\prime}\left(x_{j}\right), x_{j} \in \mathfrak{K}_{j}, j=1,2$. Consider the set of Cauchy sequences relative to both $\tau^{(j)}$ :

$$
\mathcal{X}=\left\{\left(x_{n}\right)_{n=1}^{\infty} ; x_{n} \in E,\left(x_{n}\right) \text { is a Cauchy sequence for both } \tau^{(j)}, j=1,2\right\} .
$$

Then each $\left(x_{n}\right) \in \mathcal{X}$ is associated with two limit elements $x^{(j)} \in \mathfrak{K}_{j}, j=1,2$. We define a (linear) isomorphism $\psi: \mathcal{X} \rightarrow \mathcal{X}$ by $\psi\left(x^{(1)}\right)=x^{(2)}$. The following property of $\psi$ due to Araki ([4, Lemma 6]) is known. 
Lemma 4.2. Consider the above isomorphism $\psi: \mathcal{X} \rightarrow \mathcal{X}$. Then

a) $\psi \uparrow_{E}=I$ (identity mapping on $\left.E\right)$,

b) $\left(\psi\left(x^{(1)}\right), \psi\left(y^{(1)}\right)\right)_{2}=\left(x^{(1)}, y^{(1)}\right)_{1}$,

where $x^{(j)}=\tau_{j}-\lim _{n \rightarrow \infty} x_{n}^{(j)}, y^{(j)}=\tau_{j}-\lim _{n \rightarrow \infty} y_{n}^{(j)}$, and $\left(x_{n}^{(j)}\right)_{n=1}^{\infty},\left(y_{n}^{(j)}\right)_{n=1}^{\infty} \in \mathcal{X}, j=1,2$.

Proof. We use ideas taken from [4, Lemma 6]. a): Consider $\left(x_{n}\right) \in \mathcal{X}$ satisfying $\tau_{1}-\lim _{n \rightarrow \infty} x_{n}=0$ and $\tau_{2}-\lim _{n \rightarrow \infty} x_{n}=x \in \mathfrak{K}_{2}$. Noticing

$$
\left|(x, w)_{2}\right|=\lim _{n \rightarrow \infty}\left|\left(x_{n}, w\right)\right| \leq p_{1}(w) \lim _{n \rightarrow \infty} p_{1}\left(x_{n}\right)=0
$$

for each $w \in E$, and using that for each $z \in \mathfrak{K}_{2}$ there is a sequence $\left(z_{n}\right)_{n=1}^{\infty}$, $z_{n} \in E$, such that $\lim _{n \rightarrow \infty} p_{2}\left(z-z_{n}\right)=0$, we obtain

$$
\begin{aligned}
\left|(x, z)_{2}\right| & \leq \lim _{n \rightarrow \infty}\left(\left|\left(x, z_{n}\right)_{2}\right|+\left|\left(x, z-z_{n}\right)_{2}\right|\right) \\
& =\lim _{n \rightarrow \infty}\left|\left(x, z-z_{n}\right)_{2}\right| \\
& \leq p_{2}(z) \lim _{n \rightarrow \infty} p_{2}\left(z-z_{n}\right) \\
& =0 .
\end{aligned}
$$

Since $\mathfrak{K}_{2},(., .)_{2}$ is non-degenerate, $x=0$ follows.

b): Consider $\left(x_{n}^{(j)}\right)_{n=1}^{\infty},\left(y_{n}^{(j)}\right)_{n=1}^{\infty} \in \mathcal{X}, j=1,2$. Notice that there are constants $0<c_{j}<\infty$ such that $p_{j}\left(y_{n}\right)<c_{j}, p_{j}\left(x_{n}\right)<c_{j}$ for all $n \in \mathbb{N}$. Since $\left(x_{n}, y_{n}\right)=$ $\left(x_{n}, y_{n}\right)_{1}=\left(x_{n}, y_{n}\right)_{2}$, and $\psi\left(x_{n}\right)=x_{n}, \psi\left(y_{n}\right)=y_{n}$ by a), we obtain

$$
\begin{aligned}
& \left|\left(x^{(1)}, y^{(1)}\right)_{1}-\left(x^{(2)}, y^{(2)}\right)_{2}\right| \leq \sum_{j=1}^{2}\left|\left(x^{(j)}, y^{(j)}\right)_{j}-\left(x_{n}, y_{n}\right)\right| \\
& =\sum_{j=1}^{2}\left|\left(x^{(j)}, y^{(j)}-y_{n}\right)_{j}+\left(x^{(j)}-x_{n}, y_{n}\right)_{j}\right| \\
& \leq \sum_{j=1}^{2}\left(p_{j}\left(x^{(j)}\right) p_{j}\left(y^{(j)}-y_{n}\right)+p_{j}\left(x^{(j)}-x_{n}\right) p_{j}\left(y_{n}\right)\right) \\
& \leq \sum_{j=1}^{2}\left(c_{j} p_{j}\left(y^{(j)}-y_{n}\right)+c_{j} p_{j}\left(x^{(j)}-x_{n}\right)\right) \\
& \rightarrow 0
\end{aligned}
$$

as $n \rightarrow \infty$, and complete the proof of $\mathrm{b})$. 
Lemma 4.2 readily implies the following criterion for deciding whether or not two Krein spaces $\mathfrak{K}_{j}, j=1,2$, are isometrically (and linearly) isomorphic when $E,(\cdot, \cdot)$ is densely embedded in them and Definition 2.1 a) applies.

Corollary 4.2. Let $E,(\cdot, \cdot)$ be a pseudo-Krein space and let $E$ be densely embedded in two Krein spaces $\mathfrak{K}_{j},(\cdot, \cdot)_{j}, j=1,2$, endowed with their canonical topologies $\tau^{(j)}$ such that Definition 1 a) applies to both of them. Then, the two Krein spaces $\mathfrak{K}_{j},(\cdot, \cdot)_{j}, j=1,2$, are isometrically isomorphic if and only if $\tau^{(1)} \uparrow_{E}=\tau^{(2)} \uparrow_{E}$.

\section{$\S 5 . \quad$ On Pre-Krein Spaces}

The present section is aimed at establishing criteria for deciding whether or not a pseudo-Krein space is a pre-Krein space.

Theorem 5.1. A pseudo-Krein space $E,(\cdot, \cdot)$ is a pre-Krein space if and only if all minimal majorants, each defined by a quadratic norm, are equivalent on $E$.

Proof. $(\Rightarrow)$ : Assume that there exist two minimal majorants $\tau_{*}^{(j)}, j=$ 1,2 , which are quadratic and satisfy

$$
\tau_{*}^{(1)} \neq \tau_{*}^{(2)} .
$$

Then, there are two Krein spaces $\mathfrak{K}^{(j)}=\widetilde{E}^{\tau_{*}^{(j)}}$ such that Definition 2.1 a) applies. Due to Corollary $4.2,(6)$ implies that $E,(\cdot, \cdot)$ is not a pre-Krein space. $(\Leftarrow)$ : Assume that $E,(\cdot, \cdot)$ is densely embedded in two Krein spaces $\mathfrak{K}^{(j)}, j=1,2$, which are endowed with their canonical topologies $\tau^{(j)}$ and not isometrically linear isomorphic. Lemma $4.1 \mathrm{a})$ and Corollary 4.2 yield respectively, that $\tau^{(j)} \uparrow_{E}$, being quadratic on $E,(\cdot, \cdot)$, are minimal majorants, and that $\tau^{(1)} \neq \tau^{(2)}$. Hence there are non-equivalent quadratic and minimal majorants on $E,(\cdot, \cdot)$. This completes the proof.

Remark. The assumption that the minimal majorants are quadratic in Theorem 5.1 cannot be dropped. That will be shown by an example in Section 6.4 .

Theorem 5.1 yields the following three sufficient criteria for a pseudo-Krein space to be a pre-Krein space.

Corollary 5.1. A pseudo-Krein space $E,(\cdot, \cdot)$ is a pre-Krein space if one of the following conditions is satisfied: 
(i) The weakest majorant $\tau^{*}$ exists on $E,(\cdot, \cdot)$.

(ii) One of the quadratic forms $Q$ and $-Q$ is lower semicontinuous relative to $\beta^{*}$.

(iii) The dual pairing $(E, E)$ is reflexive.

Proof. Since $E,(\cdot, \cdot)$ is a pseudo-Krein space there are quadratic minimal majorants on $E,(\cdot, \cdot)$ by Theorem $4.1,(\mathrm{i}) \Rightarrow($ ii). Condition (i) under consideration now implies that all minimal and quadratic majorants are equivalent on $E$. Hence, $E,(\cdot, \cdot)$ is a pre-Krein space by Theorem 5.1. Since (ii) $\Rightarrow(i)$ holds by Lemma $3.2 \mathrm{~d}$ ) and (iii) $\Rightarrow$ (i) by Proposition 3.1 ((i) $\Rightarrow($ iv $)$ ) and Corollary 3.1, the proof is complete.

By using results due to Wittstock, much more can be shown under the assumption that the inner product space under consideration is decomposable.

Proposition 5.1. If $E,(\cdot, \cdot)$ is a non-degenerate and decomposable inner product space, the following are equivalent:

(i) $E,(\cdot, \cdot)$ is a pre-Krein space.

(ii) The weakest majorant $\tau^{*}$ exists on $E,(\cdot, \cdot)$.

(iii) All minimal quadratric majorants of $E,(\cdot, \cdot)$ are equivalent.

(iv) One of the quadratic forms $Q$ and $-Q$ is lower semicontinuous relative to $\beta^{*}$.

(v) For every fundamental decomposition $E=E^{(+)}(\dot{+}) E^{(-)}, \beta \uparrow_{E^{(+)}}=\tau_{J} \uparrow_{E^{(+)}}$ or $\beta \uparrow_{E^{(-)}}=\tau_{J} \uparrow_{E^{(-)}}$, (where $J=P^{+}-P^{-}$, and $P^{ \pm}$are fundamental projections $\left.P^{ \pm}: E \rightarrow E^{( \pm)}\right)$.

(vi) There exists a fundamental decomposition $E=E_{1}^{(+)}(\dot{+}) E_{1}^{(-)}$such that $\beta \uparrow_{E_{1}^{(+)}}=\tau_{J_{1}} \uparrow_{E_{1}^{(+)}}$or $\beta \uparrow_{E_{1}^{(-)}}=\tau_{J_{1}} \uparrow_{E_{1}^{(-)}}$, (where $J_{1}=P_{1}^{+}-P_{1}^{-}$, and $P_{1}^{ \pm}$ are fundamental projections $: E \rightarrow E_{1}^{( \pm)}$,)

(vii) For every fundamental decomposition $E=E^{(+)}(\dot{+}) E^{(-)}, E^{(+)}\left[\tau_{J} \uparrow_{E^{(+)}}\right]$ or $E^{(-)}\left[\tau_{J} \uparrow_{E^{(-)}}\right]$is barrelled.

(viii) There exists a fundamental decomposition $E=E_{1}^{(+)}(\dot{+}) E_{1}^{(-)}$such that $E_{1}^{(+)}\left[\tau_{J_{1}} \uparrow_{E_{1}^{(+)}}\right]$or $E_{1}^{(-)}\left[\tau_{J_{1}} \uparrow_{E_{1}^{(-)}}\right]$is barrelled. 
(ix) For every fundamental decomposition $E=E^{(+)}(\dot{+}) E^{(-)}, E^{(+)}\left[\tau_{J} \uparrow_{E^{(+)}}\right]$ or $E^{(-)}\left[\tau_{J} \uparrow_{E^{(-)}}\right]$is complete, and thus a Hilbert space.

(x) There exists a fundamental decomposition $E=E_{1}^{(+)}(\dot{+}) E_{1}^{(-)}$such that $E_{1}^{(+)}\left[\tau_{J_{1}} \uparrow_{E_{1}^{(+)}}\right]$or $E_{1}^{(-)}\left[\tau_{J_{1}} \uparrow_{E_{1}^{(-)}}\right]$is complete, and thus a Hilbert space.

Proof. The equivalence of (ii), ..,(viii) is shown in [34, Satz 9]. (v) $\Rightarrow$ (ix): Notice that $E^{( \pm)},(\cdot, \cdot)_{ \pm}$are pre-Hilbert spaces, where

$$
(\cdot, \cdot)_{ \pm}= \pm(\cdot, \cdot) \Gamma_{E^{( \pm)} \times E^{( \pm)}} \cdot
$$

Consider then the dual pairings $\left(E^{( \pm)}, E^{( \pm)}\right)_{ \pm}$and notice that their Mackey topologies satisfy $\mu_{ \pm}=\tau_{J} \uparrow_{E^{( \pm)}}$. If (v) holds, Proposition 1 yields the quasicompleteness of $E^{(+)}\left[\sigma_{+}\right]$or $E^{(-)}\left[\sigma_{-}\right]$, where $\sigma_{ \pm}$denote the weak topologies of the corresponding dual pairings above. In the case that $E^{(+)}\left[\sigma_{+}\right]$is quasicomplete, consider the $\mu_{+}$-defining 0-neighborhood

$$
U=\left\{x \in E^{(+)} ;(x, x) \leq 1\right\}
$$

and notice that $U$ is closed relative to $\sigma_{+}$. The completeness of $E^{(+)}$relative to $\mu_{+}$follows now from $\sigma_{+} \prec \mu_{+}$and [18, Section 18.4 (4)]. If $E^{(-)}\left[\sigma_{-}\right]$is quasicomplete, then analogous considerations yield that $E^{(-)}\left[\mu_{-}\right]$is complete. (ix) $\Rightarrow(\mathrm{x})$ : is trivial. (x) $\Rightarrow$ (viii): If (x) holds, $E_{1}^{(+)}\left[\tau_{J_{1}} \uparrow_{E_{1}^{(+)}}\right]$or $E_{1}^{(-)}\left[\tau_{J_{1}} \uparrow_{E_{1}^{(-)}}\right]$is a Hilbert space, and thus barrelled (see [18, Section $21.5(3)]$ ). Finally, (i) $\Leftrightarrow($ iii) follows from Corollary 4.1 and Theorem 5.1.

\section{Remark.}

a) While examples of decomposable as well as non-decomposable pseudo-Krein spaces which are not pre-Krein are given in Section 6, it is not known to the author whether or not there exist non-decomposable pre-Krein spaces.

b) The assertion of Proposition $5.1(\mathrm{x}) \Rightarrow(\mathrm{ii})$ is contained also in [8, Theorem IV. 6.2].

\section{$\S 6 . \quad$ Some Examples}

For illustrating the preceding results, let us consider the following examples. Let us mention that the examples investigated in Sections 6.2 and 6.3 are related to those considered in [8, Examples IV. 4.4 and I. 11.3]. 


\section{$\S 6.1$ A pre-Krein space}

Let us construct a pre-Krein space such that Remark b) to Definition 2.1 does not apply. Consider the Hilbert space

$$
\ell_{2}=\left\{x=\left(x_{i}\right)_{i=0}^{\infty} ; \sum_{i=0}^{\infty}\left|x_{i}\right|^{2}<\infty\right\}
$$

with scalar product $[x, y]_{2}=\sum_{i=0}^{\infty} \bar{x}_{i} y_{i}$, and introduce an indefinite inner product

$$
(x, y)=[x, \mathbb{J} y]_{2},
$$

$x, y \in \ell_{2}$, where $\mathbb{J}=\operatorname{diag}(1,-1,1,-1, \ldots)$. Here diag $(\cdots)$ denotes the diagonal matrix with indicated diagonal elements. The inner product space $\ell_{2},(\cdot, \cdot)$ is non-degenerate and decomposable, and a fundamental decomposition is given by

$$
\ell_{2}=\ell_{2}^{+}(\dot{+}) \ell_{2}^{-}
$$

where

$$
\begin{aligned}
& \ell_{2}^{+}=\left\{x \in \ell_{2} ; x_{2 n+1}=0, n=0,1,2, \ldots\right\}, \\
& \ell_{2}^{-}=\left\{x \in \ell_{2} ; x_{2 n}=0, n=0,1,2, \ldots\right\} .
\end{aligned}
$$

Further, the fundamental symmetry belonging to (8) is given by

$$
\mathbb{J}=P^{+}-P^{-},
$$

where $P^{ \pm}: \ell_{2} \rightarrow \ell_{2}^{ \pm}$denote the canonical projections. Consider further the sequence space

$$
d=\left\{x \in \ell_{2} ; \text { there is an } N_{x} \in \mathbb{N} \text { such that } x_{n}=0 \text { for all } n \geq N_{x}\right\} .
$$

Consider

$$
E_{1}=\left\{x \in \ell_{2} ; P^{+}(x) \in d, P^{-}(x) \in \ell_{2}\right\}
$$

endowed with the above inner product $(\cdot, \cdot)$. A fundamental decomposition $E_{1}=E_{1}^{(+)}(\dot{+}) E_{1}^{(-)}$is then given by $E_{1}^{(+)}=d, E_{1}^{(-)}=\ell_{2}$. Notice then that $\tau_{\text {int }}\left(E_{1}^{( \pm)}\right)$is equivalent with the $\ell_{2}$-topology inherited on the linear subspaces $E_{1}^{( \pm)}$. Since $E_{1}^{(+)}$is not $\tau_{\text {int }}\left(E_{1}^{(+)}\right)$-complete, $E_{1},(\cdot, \cdot)$ is not a Krein space. On the other hand, the $\tau_{\text {int }}\left(E_{1}^{(-)}\right)$-completeness of $E_{1}^{(-)}$implies that $E_{1},(\cdot, \cdot)$ is a pre-Krein space due to Proposition 5.1. 


\section{§6.2. A decomposable pseudo-Krein space}

Using the notations introduced in Section 6.1, let us consider the transformation of coordinates given by

$$
\xi=\mathbb{U} x
$$

where $\mathbb{U}=\operatorname{diag}(U, U, \ldots)$,

$$
U=\frac{1}{\sqrt{2}}\left(\begin{array}{rr}
1 & -1 \\
1 & 1
\end{array}\right),
$$

$x \in \ell_{2}$. Notice that $\mathbb{U}$ is unitary on $\ell_{2},[\cdot, \cdot]_{2}$.

The proofs of the following lemmas are straightforward and therefore omitted.

Lemma 6.1. If $\xi=\mathbb{U} x$ and $\eta=\mathbb{U} y$, then

$$
(x, y)=[\xi, \tilde{\mathbb{J}} \eta]_{2},
$$

where $\tilde{\mathbb{J}}=\operatorname{diag}(\tilde{J}, \tilde{J}, \ldots), \tilde{J}=\left(\begin{array}{ll}0 & 1 \\ 1 & 0\end{array}\right)$.

Let us consider the vector space $E_{2}=d$ endowed with the inner product $(\cdot, \cdot)$ given in $(7)$. Notice that $E_{2},(\cdot, \cdot)$ is decomposable, and that a fundamental decomposition is given by

$$
E_{2}=d^{+}(\dot{+}) d^{-},
$$

where $d^{ \pm}=d \cap \ell_{2}^{ \pm}$.

In order to show that the example under consideration is not a pre-Krein space, let us define a family of scalar products on $E_{2}$. Introduce the following set of sequences

$$
A=\left\{\left(\alpha_{s}\right)_{s=0}^{\infty} ; \alpha_{s}>0, \varlimsup_{s \rightarrow \infty} \alpha_{s}^{-1}<\infty\right\}
$$

where $\varlimsup$ lim denotes the upper limit of $\cdot$. For each sequence $\left(\alpha_{s}\right) \in A$, introduce the matrix

$$
\begin{aligned}
\mathbb{B}_{(\alpha)} & =\operatorname{diag}\left(B_{(\alpha)}^{(0)}, B_{(\alpha)}^{(1)}, B_{(\alpha)}^{(2)}, \ldots\right), \\
B_{(\alpha)}^{(s)}=\left(\begin{array}{rr}
\alpha_{s} & 0 \\
0 & \alpha_{s}^{-1}
\end{array}\right), s= & , 1, \ldots \text {. Let us then put } \\
\langle x, y\rangle_{(\alpha)} & :=\left[\mathbb{U} x, \mathbb{B}_{(\alpha)} \mathbb{U} y\right]_{2} \\
& =\sum_{s=0}^{\infty}\left(\alpha_{s} \bar{\xi}_{2 s} \eta_{2 s}+\left(\alpha_{s}\right)^{-1} \bar{\xi}_{2 s+1} \eta_{2 s+1}\right),
\end{aligned}
$$


$\xi=\mathbb{U} x, \eta=\mathbb{U} y, x, y \in E_{2}$. Noticing that $\langle x, y\rangle_{(\alpha)}$ are scalar products on $E_{2}$, consider also the norms

$$
\|\cdot\|_{(\alpha)}=\sqrt{\langle\cdot, \cdot\rangle_{(\alpha)}}
$$

on $E_{2}$. Let $\tau_{(\alpha)}$ denote the l.c. topology defined by the norm $\|\cdot\|_{(\alpha)}$ on $E_{2}$, and

$$
\mathfrak{K}_{(\alpha)}:={\widetilde{E_{2}}}^{\tau_{(\alpha)}}=\left\{x \in \omega ;\left\{\left(\sqrt{\alpha_{s}}\right)^{-1} \xi_{2 s}\right\}_{s=0}^{\infty} \in \ell_{2},\left(\sqrt{\alpha_{s}} \xi_{2 s+1}\right)_{s=0}^{\infty} \in \ell_{2}\right\}
$$

be the $\tau_{(\alpha)}$-completed hull, where $\omega$ denotes the set of all sequences of complex numbers. In the following, let the l.c. topology defined by the continuous extension of $\|\cdot\|_{(\alpha)}$ onto $\mathfrak{K}_{(\alpha)}$ be also denoted by $\tau_{(\alpha)}$.

Lemma 6.2. Let $(\alpha),(\beta) \in A$. The following statements hold:

a) $\tau_{(\alpha)}=\tau_{(\beta)}$, if and only if $\varlimsup_{s \rightarrow \infty}\left(\alpha_{s} / \beta_{s}\right)<\infty, \varlimsup_{s \rightarrow \infty}\left(\beta_{s} / \alpha_{s}\right)<\infty$.

b) If $\tau_{(\alpha)} \neq \tau_{(\beta)}$, then $\tau_{(\alpha)}$ and $\tau_{(\beta)}$ are not comparable (i.e., neither $\tau_{(\alpha)} \succ \tau_{(\beta)}$ nor $\tau_{(\beta)} \succ \tau_{(\alpha)}$ holds).

c) $\tau_{(\alpha)}$ are minimal and quadratic majorants on $E_{2},(\cdot, \cdot)$.

Lemma 6.3. a) For each $(\alpha),(\beta) \in A$, the Gram operator $\mathbb{G}_{(\alpha, \beta)}$ satisfying

$$
\left\langle x, \mathbb{G}_{(\alpha, \beta)} y\right\rangle_{(\alpha)}=\langle x, y\rangle_{(\beta)},
$$

$x, y \in E_{2}$, is given by $\mathbb{G}_{(\alpha, \beta)}=\operatorname{diag}\left(G_{(\alpha, \beta)}^{(0)}, G_{(\alpha, \beta)}^{(1)}, G_{(\alpha, \beta)}^{(2)}, \ldots\right)$, where

$$
G_{(\alpha, \beta)}^{(s)}=\frac{1}{2}\left(\begin{array}{ll}
\left(\beta_{s} / \alpha_{s}\right)+\left(\alpha_{s} / \beta_{s}\right) & \left(\alpha_{s} / \beta_{s}\right)-\left(\beta_{s} / \alpha_{s}\right) \\
\left(\alpha_{s} / \beta_{s}\right)-\left(\beta_{s} / \alpha_{s}\right) & \left(\beta_{s} / \alpha_{s}\right)+\left(\alpha_{s} / \beta_{s}\right)
\end{array}\right),
$$

$s=0,1,2, \ldots$.

b) Furthermore, $\left(\mathbb{G}_{(\alpha, \beta)}\right)^{-1}=\mathbb{G}_{(\beta, \alpha)}, \mathbb{G}_{(\alpha, \beta)}^{*(\alpha)}=\mathbb{G}_{(\alpha, \beta)}, \mathbb{G}_{(\alpha, \beta)} \mathbb{G}_{(\beta, \gamma)}=\mathbb{G}_{(\alpha, \gamma)}$, $(\alpha),(\beta),(\gamma) \in A,\left(.^{*}(\alpha)\right.$ denotes the adjoint operator relative to $\left.\langle\cdot, \cdot\rangle_{(\alpha)}\right)$.

c) $\left.\mathbb{G}_{(\alpha, \beta)}\right) \geq 0$ on Hilbert space $\mathfrak{K}_{(\alpha)},\langle\cdot, \cdot\rangle_{(\alpha)}$.

Proposition 6.1. For each $(\alpha) \in A, \mathfrak{K}_{(\alpha)}$ is a Krein space with the fundamental symmetry

$$
\begin{aligned}
\mathbb{J}_{(\alpha)} & =\operatorname{diag}\left(J_{(\alpha)}^{(0)}, J_{(\alpha)}^{(1)}, J_{(\alpha)}^{(2)}, \ldots\right), \\
J_{(\alpha)}^{(s)} & =\frac{1}{2}\left(\begin{array}{ll}
\alpha_{s}+\alpha_{s}^{-1} & -\left(\alpha_{s}-\alpha_{s}^{-1}\right) \\
\alpha_{s}-\alpha_{s}^{-1} & -\left(\alpha_{s}+\alpha_{s}^{-1}\right)
\end{array}\right),
\end{aligned}
$$


$s=0,1,2, \ldots$, such that $(x, y)=\left\langle x, \mathbb{J}_{(\alpha)} y\right\rangle_{(\alpha)}, x, y \in \mathfrak{K}_{(\alpha)}$. Furthermore,

$$
\mathbb{J}_{(\beta)}=\mathbb{G}_{(\beta, \alpha)} \mathbb{J}_{(\alpha)},
$$

$(\alpha),(\beta) \in A$.

Proof. Considering $\varepsilon=(1,1, \ldots) \in A$ and solving the matrix equation

$$
\mathbb{G}_{(\varepsilon, \alpha)} \mathbb{J}_{(\alpha)}=\mathbb{K}_{(\alpha)} \mathbb{G}_{(\varepsilon, \alpha)},
$$

we obtain $\mathbb{K}_{(\alpha)}=\operatorname{diag}\left(K_{(\alpha)}^{(0)}, K_{(\alpha)}^{(1)}, \ldots\right)$, where

$$
K_{(\alpha)}^{(s)}=\frac{1}{2}\left(\begin{array}{rr}
a_{s} & b_{s} \\
-b_{s} & -a_{s}
\end{array}\right),
$$

$a_{s}=\alpha_{s}+\alpha_{s}^{-1}, b_{s}=\alpha_{s}-\alpha_{s}^{-1}, s=0,1,2, \ldots$. Due to $\mathbb{K}_{(\alpha)}^{*}=\mathbb{J}_{(\alpha)},(1.78)$ implies

$$
\begin{aligned}
\left\langle u, \mathbb{J}_{(\alpha)} v\right\rangle_{(\alpha)} & =\left[u, \mathbb{G}_{(\varepsilon, \alpha)} \mathbb{J}_{(\alpha)} v\right]_{2}=\left[\mathbb{K}_{(\alpha)}^{*} u, \mathbb{G}_{(\varepsilon, \alpha)} v\right]_{2} \\
& =\left\langle\mathbb{J}_{(\alpha)} u, v\right\rangle_{(\alpha)},
\end{aligned}
$$

$u, v \in E$. A simple calculation shows

$$
\left(\mathbb{J}_{(\alpha)}\right)^{2}=\mathbb{I} \quad \text { (unit matrix). }
$$

Due to (10) and (11), $\mathbb{J}_{(\alpha)}$ extends to a symmetry on $\mathfrak{K}_{(\alpha)}$, also denoted by $\mathbb{J}_{(\alpha)}$ (i.e., $\left.\mathbb{J}_{(\alpha)}=\mathbb{J}_{(\alpha)}^{*(\alpha)}=\mathbb{J}_{(\alpha)}^{-1}\right)$. Due to

$$
G_{(\varepsilon, \alpha)}^{(s)} J_{(\alpha)}^{(s)}=\left(\begin{array}{rr}
1 & 0 \\
0 & -1
\end{array}\right),
$$

$s=0,1,2, \ldots, \mathbb{G}_{(\varepsilon, \alpha)} \mathbb{J}_{(\alpha)}=\mathbb{J}$ and

$$
\left\langle x, \mathbb{J}_{(\alpha)} y\right\rangle_{(\alpha)}=\left[x, \mathbb{G}_{(\varepsilon, \alpha)} \mathbb{J}_{(\alpha)} y\right]_{2}=[x, \mathbb{J} y]_{2}=(x, y),
$$

$x, y \in \mathfrak{K}_{(\alpha)}$. This completes the proof of the first half of the proposition under consideration. The remainder follows by simple manipulation with matrices.

Corollary 6.1. a) A fundamental decomposition of $\mathfrak{K}_{(\alpha)}$ is given by

$$
\mathfrak{K}_{(\alpha)}=\mathfrak{K}_{(\alpha)}^{+}(\dot{+}) \mathfrak{K}_{(\alpha)}^{-},
$$

where $\mathfrak{K}_{(\alpha)}^{ \pm}=P_{(\alpha)}^{ \pm}\left(\mathfrak{K}_{(\alpha)}\right), P_{(\alpha)}^{ \pm}=1 / 2\left(\mathbb{I} \pm \mathbb{J}_{(\alpha)}\right)$. 
b) If $\tau_{(\alpha)} \neq \tau_{(\beta)},(\alpha),(\beta) \in A$, holds on $E_{2}$, then there is no isometrically linear isomorphism $\mathfrak{j}$ between the Krein spaces $\mathfrak{K}_{(\alpha)}$ and $\mathfrak{K}_{(\beta)}$ such that $\mathfrak{j}$ leaves each element of $E_{2}$ invariant.

Remark.

a) Note first that $E_{2},(\cdot, \cdot)$ is a pseudo-Krein space by Proposition 6.1. Notice then that, due to Lemma $6.2 \mathrm{a})$, there are $(\alpha),(\beta) \in A$ such that $\tau_{(\alpha)} \neq \tau_{(\beta)}$ on $E_{2}$. Due to Corollary 4.1 this implies that $E_{2},(\cdot, \cdot)$ is not a pre-Krein space.

b) It is easily seen that if $\tau_{(\alpha)} \neq \tau_{(\beta)}$, then $\sqrt{G_{\alpha, \beta}}$ is an isometrical isomorphism between the Krein spaces $\mathfrak{K}_{(\alpha)}$ and $\mathfrak{K}_{(\beta)}$, which however does not leave some elements of $E$ invariant.

Let us now consider the dual pairing

$$
\left(E_{2}, E_{2}\right)
$$

and its l.c. topologies recalled in Section 3.1, where the duality is given by the inner product (7). Notice that the weak topology $\sigma$ is given by the system of seminorms

$$
\mathfrak{P}(\sigma)=\left\{x \rightarrow p_{y}(x)=|(x, y)| ; y \in E_{2}\right\},
$$

$x \in E_{2}$. It follows that a set $B \subset E_{2}$ is $\sigma$-bounded, if and only if $\sup _{b \in B}\left\{\left|b_{i}\right|\right\}=$ $c_{i}<\infty, i=0,1,2, \ldots$, where $b=\left(b_{0}, b_{1}, \ldots\right)$. Hence, the strong topology $\beta$ is defined by the system of seminorms

$$
\mathfrak{P}(\beta)=\left\{x \rightarrow p_{(c)}(x):=\sum_{i=0}^{\infty} c_{i}\left|x_{i}\right| ;\left(c_{i}\right)_{i=0}^{\infty} \in \mathbb{R}_{+}^{\mathbb{N}}\right\},
$$

$x \in E_{2}$, and $\mathbb{R}_{+}^{\mathbb{N}}$ denotes the set of all sequences of positive reals. It now follows that $D \subset E_{2}$ is $\beta$-bounded if and only if there are $n_{D} \in \mathbb{N}$ such that $a_{m}=0, m=n_{D}+1, n_{D}+2, \ldots$, for any $a=\left(a_{0}, a_{1}, \ldots\right) \in D$, and furthermore

$$
\sup _{a \in D}\left\{\left|a_{i}\right|\right\}=c_{i}<\infty
$$

$i=0,1, \ldots, n_{D}$. The topology $\beta^{*}$ is thus defined by the system of seminorms

$$
\mathfrak{P}\left(\beta^{*}\right)=\left\{x \rightarrow q_{(c)}(x):=\sum_{i=0}^{m} c_{i}\left|x_{i}\right| ; m \in \mathbb{N},\left(c_{i}\right)_{i=0}^{\infty} \in \mathbb{R}_{+}^{\mathbb{N}}\right\} .
$$

Obviously, $\sigma=\beta^{*}$ and a defining system of seminorms is also given by

$$
\mathfrak{P}(\sigma)=\left\{x \rightarrow\left|x_{i}\right| ; i=0,1,2, \ldots\right\} .
$$

The following is now obvious. 
Lemma 6.4. $\quad$ For every $(\alpha) \in A$, it follows that $\sigma=\beta^{*} \supsetneqq \tau_{(\alpha)} \supsetneqq \beta$.

\section{$\S 6.3$ A non-decomposable pseudo-Krein space}

\section{Consider}

$E_{3}=\left\{x \in \ell_{2} ;\right.$ there is an $N_{x} \in \mathbb{N}$ such that $x_{2 n}=x_{2 n+1}$ for all $\left.n \geq N_{x}\right\}$ endowed with the inner product $(\cdot, \cdot)$ introduced on $\ell_{2}$ in $(7)$. Notice that

$$
E_{2} \varsubsetneqq E_{3} \varsubsetneqq \ell_{2} \text {. }
$$

Proposition 6.2. a) The inner product space $E_{3},(\cdot, \cdot)$ is non-degenerate and non-decomposable.

b) For each $(\alpha) \in A$, the norm $\|\cdot\|_{(\alpha)}$ continuously extends to $E_{3}$, and $\mathfrak{K}_{(\alpha)}=$ ${\widetilde{E_{3}}}^{\tau_{(\alpha)}}$ holds.

c) $E_{3}=\bigcap_{\alpha \in A} \mathfrak{K}_{(\alpha)}$.

Proof. a) $E$ is non-degenerate, since for each non-zero $x=\left(x_{i}\right)_{i=0}^{\infty} \in E$ there are $x_{i_{0}} \neq 0$ and $y=\left(\delta_{i_{0}, n}\right)_{n=0}^{\infty} \in E$ such that $|(x, y)|=\left|x_{i_{0}}\right| \neq 0$. Setting

$$
L_{ \pm}=\left\{x \in E_{3} ; x_{2 \nu}= \pm x_{2 \nu+1}, \nu=0,1, \ldots\right\}
$$

notice that $E_{3}=L_{+} \dot{+} L_{-}$(algebraic direct sum), $L_{ \pm} \subset \mathcal{P}^{0}\left(E_{3}\right)$, and $L_{+} \cong d$,

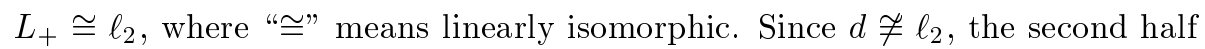
of a) follows from [7, Theorem I.1.34]. b): For each $x=\left(x_{s}\right)_{s=0}^{\infty} \in E_{3}$, consider the sequence $\left\{x^{(n)}\right\}_{n=1}^{\infty}, x^{(n)}=\left(x_{s}^{(n)}\right)_{s=0}^{\infty} \in E_{2}$, where

$$
x_{s}^{(n)}=\left\{\begin{array}{rl}
x_{s} & \text { for } \quad s<2 n \\
0 & \text { for } \quad s \geq 2 n
\end{array} .\right.
$$

Noticing that $(\alpha) \in A$ implies the existence of some constant $0<c<\infty$ such that $\alpha_{s}^{-1}<c$ for all $s=0,1,2, \ldots$, we obtain for $n>N_{x}$

$$
\begin{aligned}
\left\|x-x^{(n)}\right\|_{(\alpha)} & =\sqrt{2} \sum_{s=2 n+1}^{\infty} \alpha_{s}^{-1}\left|x_{2 s+1}\right|^{2} \leq c \sqrt{2} \sum_{s=2 n+1}^{\infty}\left|x_{2 s+1}\right|^{2} \\
& \rightarrow 0
\end{aligned}
$$


as $n \rightarrow \infty$, where $N_{x}$ is taken from the above definition of $E_{3}$, and $(\mathbb{U} x)_{2 s+1}=$ $\sqrt{2} x_{2 s+1},(\mathbb{U} x)_{2 s}=0, s \geq N, x \in E_{3} \subset \ell_{2}$ are used. Further

$$
\begin{aligned}
\|x\|_{(\alpha)} & =\sum_{s=0}^{\infty}\left(\alpha_{s}\left|\xi_{2 s}\right|^{2}+\alpha_{s}^{-1}\left|\xi_{2 s+1}\right|^{2}\right) \\
& \leq \sum_{s=0}^{N_{x}} \alpha_{s}\left|\xi_{2 s}\right|^{2}+c \sum_{s=0}^{\infty}\left|\xi_{2 s+1}\right|^{2}<\infty
\end{aligned}
$$

for every $x \in E_{3},(\alpha) \in A$. Hence, ${\widetilde{E_{2}}}^{\tau_{(\alpha)}} \supset E_{3}$. Using (12), (16), we obtain

$$
{\widetilde{E_{3}}}^{\tau_{(\alpha)}}={\widetilde{E_{2}}}^{\tau_{(\alpha)}}=\mathfrak{K}_{(\alpha)},
$$

proving b). c): Using b) and noticing that $\bigcap_{(\alpha) \in A} \mathfrak{K}_{(\alpha)}$ is well-defined due to Araki's lemma (cf. Lemma 4.2), we obtain $E_{3} \subset \bigcap_{(\alpha) \in A} \mathfrak{K}_{(\alpha)}$. Assume now that there is an element $y \in\left(\bigcap_{(\alpha) \in A} \mathfrak{K}_{(\alpha)}\right) \backslash E_{3}$. Again with the help of transformation (10), the relation given in the definition of $E_{3}$ readily yields

$$
E_{3}=\left\{x \in \ell_{2} ; \text { there is an } N_{x} \text { with } \xi_{2 n}=0, n=N_{x}, N_{x}+1, \ldots\right\} .
$$

Recalling $\mathfrak{K}_{(\varepsilon)}=\ell_{2}$, we have $y \in \ell_{2}$. Further, for $\eta=\left(\eta_{0}, \eta_{1}, \ldots\right), \eta=\mathbb{U} y$, $y \in \ell_{2} \backslash E_{3}$, there is a subsequence $\left(\eta_{n_{s}}\right)_{s=1}^{\infty}$ with $\eta_{n_{s}} \neq 0, n_{1}<n_{2}<\cdots$. Considering now $\left(\beta_{n}\right)_{n=0}^{\infty} \in A$ with $\beta_{n_{s}}=\left|\eta_{n_{s}}\right|^{-1}, s=1,2, \ldots$, we conclude that $\|y\|_{(\beta)}=\infty$. Hence, $y \notin \mathfrak{K}_{(\beta)}$.

Remark. The above Proposition 6.2 readily implies that $E_{3}(\cdot, \cdot)$ is a pseudo-Krein but not a pre-Krein space.

\section{§6.4. The Araki-Hansen example}

The following example is due to Araki and Hansen (see [12, Example 2.10]). Consider the (complex) sequence space

$$
E_{4}=\left\{x=\left(x_{0}, x_{1}, \ldots\right) \in \omega ; \sum_{n=1}^{\infty} n^{2}\left|x_{n}\right|^{2}<\infty\right\}
$$

endowed with the inner product

$$
(x, y)_{4}=\sum_{i, j=1}^{\infty} \varepsilon_{i j} \bar{x}_{i} y_{j},
$$


where

$$
\varepsilon_{i j}=\left\{\begin{aligned}
(-1)^{(j)_{i}} & \text { for } i \leq j \\
\varepsilon_{j i} & \text { for } i>j
\end{aligned}\right.
$$

and $(j)_{i} \in\{0,1\}$ is taken from the binary expansion $j=\sum_{m=0}^{\infty}(j)_{m} 2^{m}$.

Setting $\|x\|_{1}=\sum_{n=1}^{\infty}\left|x_{n}\right|,\|x\|=\sqrt{\sum_{n=1}^{\infty} n^{2}\left|x_{n}\right|^{2}}$, it follows

$$
\begin{aligned}
\|x\|_{1}{ }^{\prime} & =\|x\|_{1} \\
\|x\|_{1} & \leq \frac{\pi}{6}\|x\|, \\
\left|(x, y)_{4}\right| & \leq\|x\|_{1}\|y\|_{1} \leq \frac{\pi}{6}\|x\|\|y\|,
\end{aligned}
$$

$x, y \in E_{4}$ (see [12, p. 898]).

Due to (17), (18), it follows that $\tau\left(\|\cdot\|_{1}\right)$ is a minimal majorant on $E_{4},(\cdot, \cdot)_{4}$. Noticing that $\|\cdot\|_{1}$ is not quadratic, we deduce the existence of minimal majorants which are not quadratic. Further, since the norm $\|\cdot\|$ is quadratic, (18) and Theorem 4.1 imply that $E_{4},(\cdot, \cdot)_{4}$ is a pseudo-Krein space. Applying Lemma 4.1 a) (or the Aronszajn-Schatten construction to the norm $\|\cdot\|)$, we deduce the existence of a minimal and quadratic majorant $\tau(p)$ on $E_{4},(\cdot, \cdot)_{4}$. Thus $\tau(p) \neq \tau\left(\|\cdot\|_{1}\right)$. This shows that the weakest majorant $\tau^{*}$ does not exist.

Due to Lemma 3.2 d), Proposition $3.1((\mathrm{i}) \Rightarrow(\mathrm{iv}))$ and Corollary 3.1, the above implies

(i) the quadratic forms $Q$ and $-Q$ are not lower semicontinuous relative to $\beta^{*}\left(E_{4}, E_{4}\right)_{4}$, where $Q(x)=(x, x)_{4}, x \in E_{4}$, and

(ii) $\left(E_{4}, E_{4}\right)_{4}$ is a non-reflexive dual pairing.

Consider now the inner product space $\ell_{1}={\widetilde{E_{4}}}^{\tau\left(\|\cdot\|_{1}\right)}$ endowed with the inner product $(\cdot, \cdot)_{4} \tilde{\text { }}$, which is obtained by $\tau\left(\|\cdot\|_{1}\right)$-continuous extension of $(\cdot, \cdot)_{4}$. Since $\ell_{1}\left[\tau\left(\|\cdot\|_{1}\right)\right]$ is barrelled, the dual pairing $\left(\ell_{1}, \ell_{1}\right)_{4}^{\sim}$ is reflexive by Proposition 3.1, and the weakest majorant $\tau^{*}=\tau\left(\|\cdot\|_{1}\right)$ exists on $\ell_{1},(\cdot, \cdot) \tilde{4}$ by Corollary 3.2. Due to Theorem $4.1, \ell_{1},(\cdot, \cdot)_{4}^{\sim}$ is not a pseudo-Krein space. Hence the assumption of Corollary 5.1 that the inner product space under consideration has to be a pseudo-Krein space cannot be dropped. Finally, $\ell_{1},(\cdot, \cdot)_{4}$ is not decomposable by Corollary 4.1 .

\section{Acknowledgements}

The author would like to thank Professor H. Araki for helpful and stimulating discussions on the subject of the present paper. The helpful comments of the referee are gratefully acknowledged. 


\section{References}

[1] Albeverio, S., Gottschalk, H. and Wu, J.-L., Euclidean random fields, pseudodifferential operators, and Wightman functions, in: I. M. Davies, A. Truman and K. D. Elworthy (eds.), Stochastic Analysis and Applications, Singapore: World Scientific 1996, pp. 2037.

[2] - Models of local relativistic quantum fields with indefinite metric (in all dimensions), Commun. Math. Phys., 184 (1997), 509-531.

[3] Antoine, J.-P. and Ôta, S., Unbounded GNS representations of a *-algebra in a Krein space, Lett. Math. Phys., 18 (1989), 267-274.

[4] Araki, H., On a pathology in indefinite inner product spaces, Commun. Math. Phys., 85 (1982), 121-128.

[5] — Indecomposable representations with invariant inner product. A theory of the Gupta-Bleuler triplet, Commun. Math. Phys., 97 (1985), 149-159.

[6] Aronszajn, N., Quadratic forms on vector spaces, Proc. Internat. Sympos. Linear Spaces, Jerusalem, 1960.

[7] Azizov, T. Y. and Iokhvidov, I. S., Linear operators in spaces with indefinite metric, John Wiley \& Sons Inc., New York, 1989.

[8] Bognár, J., Indefinite inner product spaces, Springer-Verlag, Berlin, 1974.

[9] Bogoljubov, N. N., Logunov, A. A., Oksak, A. I. and Todorov, I. T., General principles of quantum field theory, Dordrecht, Kluwer, 1990.

[10] Borchers, H. J., On the structure of the algebra of field operators, Nuovo Cimento, 24 (1962), 214-236.

[11] Constantinescu, T. and Gheondea, A., Representations of Hermitian kernels by means of Krein spaces, II. Invariant kernels, preprint Bucuresti, nr 4/2000.

[12] Hansen, F., Selfpolar norms on an indefinite inner product space, Publ. RIMS, Kyoto University, 16 (1980), 889-913.

[13] Hofmann, G., On the GNS representation of generalized free fields with indefinite metric, Rep. Math. Phys., 38 (1996), 67-84.

[14] — Generalized free field like $U(1)$-gauge theories within the Wightman framework, Rep. Math. Phys., 38 (1996), 85-103.

[15] - On GNS representations on indefinite inner product spaces, I. The structure of the representation space, Commun. Math. Phys., 191 (1998), 299-323.

[16] Jakobczyk, L. and Strocchi, F., Euclidean formulation of quantum field theory without positivity, Commun. Math. Phys., 119 (1988), 529-541.

[17] Jarchow, H., Locally Convex Spaces, B. G. Teubner-Verlag, Stuttgart, 1981.

[18] Köthe, G., Topological vector spaces, I. Springer-Verlag, Berlin, New York, Heidelberg, 1984.

[19] Kugo, T. and Ojima, I., Local covariant operator formalism of non-abelian gauge theories and quark confinement problem, Suppl. of the Progr. Theor. Phys., 66 (1979), 1-130.

[20] Morchio, G. and Strocchi, F., Infrared singularities, vacuum structure and pure phases in local quantum field theory, Ann. Inst. Henri Poincaré, 33 (1980), 251-282.

[21] Morchio, G., Pierotti, D. and Strocchi, F., Infrared and vacuum structure in twodimensional local quantum field theory models. The massless scalar field, J. Math. Phys., 31 (1990), 1467-1477.

[22] Nakagami, Y., Tomita's spectral analysis in Krein spaces, Publ. RIMS, Kyoto Univ., 22 (1986), 637-658.

[23] — Spectral analysis in Krein spaces, Publ. RIMS, Kyoto Univ., 24 (1988), 361-378.

[24] Nakagami, Y. and Tomita, M., Triangular matrix representation for selfadjoint operators in Krein spaces, Japan. J. Math., 14 (1988), 165-201.

[25] Nakanishi, N. and Ojima, I., Covariant operator formalism of gauge theories and quantum gravity, Singapore, World Scientific, 1990.

[26] Neumark, M. A., Normierte Algebren, Verlag Harri Deutsch, Thun, Frankfurt am Main, 1990. 
[27] Nevanlinna, R., Über metrische linear Räume. II. Bilinearformen und Stetigkeit, Ann. Acad. Sci. Fenn., AI 113 (1952).

[28] Ôta, S., Unbounded representation of a *-algebra on indefinite metric space, Ann. Inst. Henri Poincaré, 48 (1988), 333-353.

[29] Scheibe, E., Über Feldtheorien in Zustandsräumen mit indefiniter Metrik, mimeographed notes of the Max-Planck Institut für Physik und Astrophysik in München, München, 1960.

[30] Strocchi, F., Selected Topics on the General Properties of QFT, Lecture Notes in Physics, 51, World Scientific, Singopore-New Jersey-Hong Kong, 1993.

[31] Tomita, M., Operators and operator algebras in Krein spaces, I. Spectral analysis in Pontrjagin spaces, Publ. RIMS, Kyoto Univ., kokyuroku, 398 (1980), 131-158.

[32] Uhlmann, A., Über die Definition der Quantenfelder nach Wightman und Haag, Wiss. Zeitschr. d. Univ. Leipzig, 11 (1962), 213-217.

[33] Wilansky, A., Modern methods in topological vector spaces, New York, 1978.

[34] Wittstock, G., Über Majoranten indefiniter Bilinearformen, Ann. Acad. Sci. Fenn., AI 381 (1966).

[35] — Über indefinit symmetrisierbare lineare Abbildungen, Math. Z., 111 (1969), 131-144.

[36] Yngvason, J., Remarks on the reconstruction theorem for field theories with indefinite metric, Rep. Math. Phys., 12 (1977), 57-64. 